\title{
Microsurgical Localization of the Cochlea in the Extended Middle Fossa Approach
}

\author{
Jonathan A. Forbes ${ }^{1}$ Alejandro Rivas ${ }^{2}$ Betty Tsai ${ }^{2}$ Moneeb Ehtesham ${ }^{1}$ Scott Zuckerman ${ }^{1}$ \\ George Wanna ${ }^{2}$ Kyle Weaver ${ }^{1}$ \\ ${ }^{1}$ Department of Neurological Surgery, Vanderbilt University Medical \\ Center, Nashville, Tennessee, United States \\ ${ }^{2}$ Department of Otolaryngology, Vanderbilt University Medical \\ Center, Nashville, Tennessee, United States \\ Address for correspondence and reprint requests Jonathan A. Forbes, \\ MD, Department of Neurological Surgery, T-4224, Medical Center \\ North, 1161 21st Ave South, Nashville, Tennessee 37232, United \\ States (e-mail: Jonathan.forbes@vanderbilt.edu).
}

J Neurol Surg B 2012;73:410-414.

\begin{abstract}
Objective In the extended middle fossa approach, a portion of the petrous bone known as Kawase's rhomboid can be drilled to expose the posterior fossa through a middle fossa corridor. During this bony resection, the cochlea is placed at risk. The objective of this study was to objectively detail the position of the cochlea in relation to reliable surgical landmarks.

Methods Eleven cadaveric specimens were dissected-including six cadaveric heads and five dry temporal bones by means of an anterior petrosectomy with skeletonization of the cochlea. Three anatomic measurements describing the location of the cochlea in relation to the extrapolated intersection of the greater superficial petrosal nerve (GSPN) and facial nerve were recorded. These measurements were then correlated with thin-cut temporal bone computed tomography scans from 25 patients with morphologically normal inner ears.

Results In the cadaveric specimens, the anterior border of the membranous basal turn of the cochlea was located an average of $7.56 \mathrm{~mm}(6.4$ to $8.9 \mathrm{~mm})$ anterior to the extrapolated junction of the GSPN and facial nerve, as measured along the course of the GSPN. The medial border of the membranous cochlea (medial margin of basal turn) was located an average of $8.2 \mathrm{~mm}$ ( 6.9 to $8.9 \mathrm{~mm}$ ) medial to the extrapolated junction of the GSPN and facial nerve, as measured along the course of the facial nerve. The average

Keywords

- middle fossa approach

- Kawase's rhomboid

- cochlea

- greater superficial petrosal nerve maximum distance from the extrapolated junction of the GSPN and facial nerve to the membranous cochlea was $9.3 \mathrm{~mm}$ ( 8.2 to $10.3 \mathrm{~mm}$ ). These anatomic measurements correlated well with radiologic measurements of the same parameters.

Conclusion When drilling Kawase's rhomboid, it is useful to locate the extrapolated junction of the GSPN and the facial nerve. Drilling of the anteromedial petrous bone outside of a radius of $12.5 \mathrm{~mm}$ from the extrapolated junction of GSPN and facial nerve appears to be associated with a low degree of risk to the cochlear apparatus.
\end{abstract}

\section{Introduction}

In the extended middle fossa approach, a portion of the anteromedial petrous bone known as Kawase's rhomboid

received

January 26, 2012

accepted after revision

June 26, 2012

published online

November 7, 2012

can be resected to provide exposure of the posterior fossa through a middle fossa corridor. Kawase's rhomboid is bordered by the petrous ridge medially, the greater superficial petrosal nerve (GSPN) and internal carotid artery (ICA)

Copyright $\odot 2012$ Georg Thieme Verlag KG Stuttgart · New York
DOI http://dx.doi.org/ 10.1055/s-0032-1329621. ISSN 2193-6331. 
laterally, the arcuate eminence posteriorly, and the V3 division of the trigeminal nerve anteriorly. ${ }^{1}$ This area, in turn, can be bisected by the internal acoustic canal (IAC) into pre- and post-meatal triangles. During bony resection of Kawase's rhomboid, the cochlea-in addition to various other structures-is placed at significant risk. Damage to the cochlea results in ipsilateral hearing loss and vertigo, and it may be associated with facial weakness if the nearby facial nerve is injured.

Minimization of risk during bony resection of the rhomboid construct is contingent on extensive knowledge of the microsurgical anatomy of the cochlea and its association with several external landmarks. This study was conducted to evaluate the relationship of the cochlea to Kawase's rhomboid and provide useful information for surgeons planning to perform an anterior petrosectomy to augment posterior fossa exposure.

\section{Methods}

\section{Cadaveric Dissection}

A total of 11 cadaveric specimens were dissected, including wet bench dissection of 6 cadaveric heads and 5 dry temporal bones. Each of the 6 cadaveric heads was positioned in pins for a standard middle fossa craniotomy. A curvilinear "question mark" incision was fashioned about $1 \mathrm{~cm}$ in front of the tragus, initially curving posteriorly then anteriorly. Burr holes were placed superior to the zygomatic arch and at the superior margin of the craniotomy. A craniotome was used to turn a $5-\mathrm{cm}$ bone flap located approximately one third behind and two thirds in front of the external auditory canal. A Leksell rongeur was then used to extend the craniotomy flush with the middle fossa floor. Subtemporal dissection was accomplished in a posterior to anterior dissection until the medial extent of the petrous ridge could be visualized. Following completion of the extradural, subtemporal dissection, the porus trigeminus could be visualized anteromedially and the arcuate eminence posteriorly.

Following subtemporal dissection, the petrous apex was drilled in the manner previously described by Miller et $\mathrm{al}^{2}$ until the dura of the seventh/eighth nerve complex could be

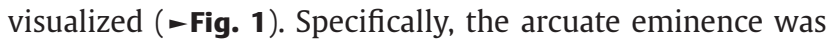
first "blue lined" (e.g., drilled until the membrane could be visualized) until the membranous superior semicircular canal (SSCC) was visible. The GSPN was identified and tracked posteriorly to its exit from the facial hiatus. The angle between the superior semicircular canal and GSPN was bisected to reveal the approximate location of the IAC. ${ }^{3}$ Drilling over the expected location of the IAC was continued until the dura of the seventh/eighth nerve complex was visualized. Drilling was then extended anterolaterally until the basal turn of the cochlea had been "blue lined"; blue lining of the cochlea was performed to improve measurement accuracy-as differentiation of the cortical bone of the cochlea from surrounding cortical bone of the petrous temporal bone is not always straightforward (blue lining the cochlea would not otherwise have been performed in a standard anterior petrosectomy). A diamond drill was then used to

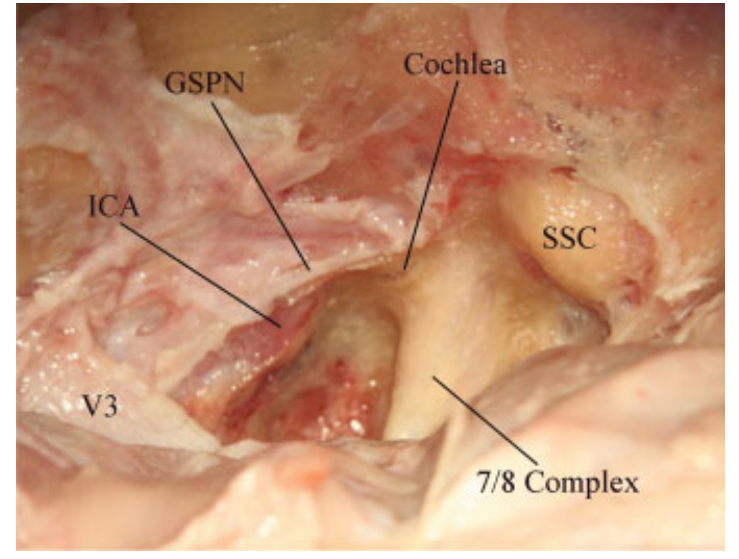

Fig. 1 Anatomy encountered in extradural extended middle fossa approach (photo obtained following dissection of cadaveric head as described in text). Left of picture is anterior, bottom of picture is medial. GSPN, greater superficial petrosal nerve; ICA, internal carotid artery; SSC, superior semicircular canal; V3, third division of trigeminal nerve.

expose the carotid artery under the GSPN. Once the seventh/ eighth nerve complex, petrous ICA, cochlea, and SSCC had been exposed, the remainder of the anterior petrosal bone was then resected.

Following completion of the steps described above, a postoperative computed tomography (CT) scan was obtained to verify skeletonization. After review and approval of the CT images, three morphologic measurements were made on the cadaveric specimen. Quantification of the following distances, as depicted in -Fig. 2, was then made with an industrial grade Starrett No. 274 3-inch caliper: (1) The extrapolated junction of the GSPN and facial nerve to the membranous anterior border of cochlea (measured along the GSPN). The anteriormost point corresponded to the anterior limit of the basal turn of the cochlea. (2) The extrapolated junction of the GSPN and facial nerve to the medial membranous border of cochlea (measured along cranial nerve $[\mathrm{CN}] 7$ ). The medialmost point corresponded to the medial margin of the basal turn of the cochlea. (3) The average maximum distance from the extrapolated junction of the GSPN and facial nerve to the basal turn of the membranous cochlea.

The procedure used in analysis of the dry temporal bones (visualized in - Fig. 2A) was similar. The GSPN was immediately visible in selected dry temporal bone specimens. Bone underneath the GSPN was drilled until the petrous ICA was visible. The IAC in these specimens could be directly visualized and was drilled until the seventh and eighth nerve complex had been completely unroofed. The drilling was extended anterolaterally until the cochlea could be identified. The above measurements were repeated following completion of the anterior petrosectomy.

It is important to mention that, although portions of the technique described on cadaveric specimens in this study are routinely used to gain access to the petrous apex, other maneuvers described (e.g., "blue-lining" the cochlea) do not represent standard surgical procedure and would introduce significant risk to eloquent structures if utilized in an 


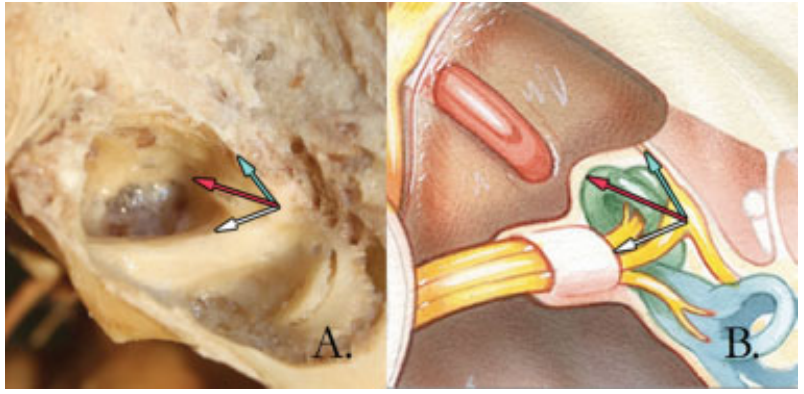

Fig. 2 Illustration of anatomic measurements that were obtained. A drilled, dry temporal bone following skeletonization (A). The green arrow depicts the distance from the extrapolated junction of the greater superficial petrosal nerve (GSPN) and facial nerve (base of the arrow) to anterior border of the basal turn of the cochlea (tip of the arrow). This distance is measured along the GSPN. The white arrow depicts the distance from the extrapolated junction of the GSPN and facial nerve (base of arrow) to the medial border of the basal turn of the cochlea (tip of the arrow). This distance is measured along the meatal trajectory of cranial nerve 7 . The red arrow depicts the maximum distance from the extrapolated junction of the GSPN and facial nerve to the basal turn of the cochlea (tip of arrow). Extrapolated junction of the GSPN and facial nerve represent the approximate location of the geniculate ganglion. Depiction of the aforementioned measurements in an illustration, courtesy of Jackler et al (B). ${ }^{12}$

intraoperative setting. Thus, the methods of cadaveric dissection described in this study should not be interpreted as standard surgical technique.

\section{Radiologic Assessment}

A cohort of 25 patients who had received thin-cut CT imaging though the temporal bones was obtained from the neurootology database. These patients underwent imaging for a variety of reasons, but all were deemed to have bilateral morphologically normal inner ears. The above measurements were repeated using standard PACS software (-Fig. $\mathbf{3}$ ). It is important to note that the position of the GSPN was estimated, in these scans, using the bony groove of the GSPN that is visible deep to this structure.

\section{Results}

- Table 1 details the anatomic measurements defining the relationship of the cochlea and adjacent structures. Special

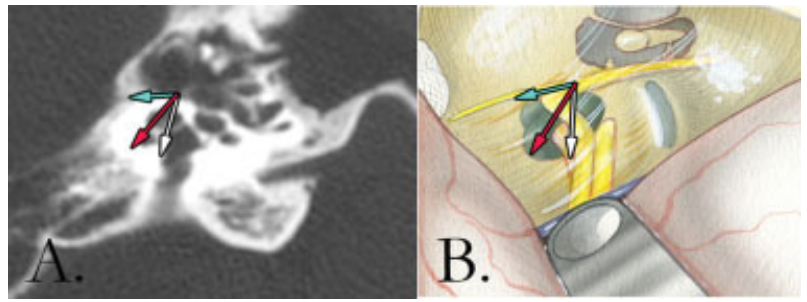

Fig. 3 Computed tomography image with greater superficial petrosal nerve (GSPN) canal, cochlea, and meatus of $7 / 8$ complex visible (A). The green arrow depicts the distance from the extrapolated junction of the GSPN and facial nerve (base of the arrow) to the anterior border of the basal turn of the cochlea (tip of the arrow). This distance is measured along the GSPN. The white arrow depicts the distance from the extrapolated junction of the GSPN and the facial nerve (base of arrow) to the medial border of the cochlea (tip of the arrow). This distance is measured along the meatal trajectory of cranial nerve 7 . The red arrow depicts the maximum distance from the extrapolated junction of the GSPN and facial nerve to the anterior edge of the basal turn of the cochlea (tip of arrow). Depiction of the aforementioned measurements in an illustration, courtesy of Jackler et al (B). ${ }^{12}$

attention is paid to the maximum distances encountered from the extrapolated junction of the GSPN and facial nerve to the membranous basal turn of the cochlea, as these figures represent the distance, outside which drilling is associated with a low degree of risk to the membranous cochlea (highlighted by red box). Review of this data indicates that drilling outside of a radius of $11 \mathrm{~mm}$ from the extrapolated junction of the GSPN and facial nerve is associated with a low degree of risk to the membranous cochlear apparatus. - Table $\mathbf{2}$ additionally details measured dimensions of cochlea obtained following review of thin-cut CT imaging in 25 patients.

\section{Discussion}

The complexity of the extended middle fossa approach relates to the close anatomic proximity of the internal acoustic canal, cochlea, semicircular canal, GSPN, and ICA. In this procedure, the anteromedial petrous bone-also known as Kawase's rhomboid-is removed to improve visualization of the posterior fossa. During removal of the Kawase's rhomboid, skull base surgeons must be aware of the location of the cochlea at all times. Previous authors have attempted to delineate the

Table 1 Measurements of extrapolated junction of greater superficial petrosal nerve and facial nerve to cochlear borders

\begin{tabular}{|l|l|l|l|l|l|l|}
\hline Measurement & Cadaveric specimen & Mean & Median & Minimum & Maximum & SD \\
\hline \multirow{2}{*}{ EJ-medial CO (white arrow) } & Cadaveric head & 8.4 & 8.3 & 6.9 & 9.0 & .766 \\
\cline { 2 - 7 } & Temporal bone & 8.0 & 8.0 & 6.9 & 8.9 & .760 \\
\hline EJ-anterior CO (green arrow) & Cadaveric head & 7.8 & 8.0 & 6.4 & 8.1 & .880 \\
\hline \multirow{2}{*}{ EJ-basal turn CO (red arrow) } & Temporal bone & 7.5 & 7.5 & 6.6 & 8.7 & .862 \\
\cline { 2 - 7 } & Cadaveric head & 9.4 & 9.4 & 8.2 & 10.3 & .776 \\
\cline { 2 - 7 } & Temporal bone & 9.3 & 9.3 & 8.3 & 10.0 \\
\hline
\end{tabular}

Abbreviations: CO, cochlea; EJ, extrapolated junction of greater superficial petrosal nerve and facial nerve (approximate location of geniculate ganglion); SD, standard deviation. 
Table 2 Cochlear measurements from computed tomography (CT) imaging

\begin{tabular}{|l|l|l|l|l|l|}
\hline Measurement & Mean & Median & Minimum & Maximum & SD \\
\hline EJ-medial CO (white arrow) & 7.9 & 7.8 & 6.3 & 9.3 & .064 \\
\hline EJ-anterior CO (green arrow) & 5.7 & 5.6 & 4.7 & 7.3 & .062 \\
\hline Ej-basal turn CO (red arrow) & 8.7 & 8.7 & 7.4 & 10.5 & .061 \\
\hline
\end{tabular}

Abbreviations: CO, cochlea; EJ, extrapolated junction of greater superficial petrosal nerve and facial nerve (approximate location of geniculate ganglion); SD, standard deviation. Note: Measured dimensions of cochlea obtained following review of thin-cut CT imaging in 25 patients. Values include measurement of 25 right and 25 left temporal bones.

location of the cochlea in relation to surgical landmarks. Diaz Day et al described a pre-meatal triangle bordered by the GSPN laterally, the IAC posteriorly, and the medial petrous ridge and porus trigeminus medially. In addition to its referencing its borders, this triangle was described by three points: the internal carotid artery genu, the geniculate ganglion, and the medial lip of the IAC. The authors reported that the cochlea could reliably be found in the lateral half of this triangle-and stressed the close proximity of the cochlea to the genu of the petrous carotid. ${ }^{1}$ In a manuscript with similar conclusions, Isolan et al reported that the cochlea could be exposed by drilling the lateral aspect of the posteromedial triangle-whose borders are defined by the porus trigeminus, cochlea, and mandibular division of the trigeminal nerve. ${ }^{4}$ Sameshima et al noted that the cochlea resides in a plane deeper than the IAC and GSPN, lateral to the genu of the ICA. ${ }^{5}$ Mastronardi et al described two theoretical "fans" with a 90-degree relationship to one other-useful in identifying the vascular, nervous, and osseous structures, including the cochlea, of Kawase's rhomboid. ${ }^{6}$ In a study describing exposure of the horizontal petrous carotid artery in preparation for intrapetrous carotid bypass, Dew et al reported the average distance between the cochlea and carotid to be $4.3 \mathrm{~mm}^{7}$ In a separate manuscript, Rhoton reported this distance to be an average of $2.1 \mathrm{~mm} .^{8}$

Two previous studies have reported data particularly relevant to this discussion. Jung et al dissected 32 cadaveric specimens and found the average distance between the cochlea and geniculate ganglion to be $3.0 \pm 0.8 \mathrm{~mm} .{ }^{9}$ Though useful, this information is limited in the sense that it does not help the skull base surgeon define the anteromedial border of the cochlea-the border placed at greatest risk during anterior petrosectomy. A second study by Sennaroglu et al provided an extensive review that included 39 anatomic measurements of the petrous temporal bone obtained from 10 cadaveric specimens. ${ }^{10}$ One of these measurements was from the IAC apex to the anteromedial cochlear boundary-roughly corresponding to the measurement (depicted by the red arrow above) from the extrapolated junction of the GSPN and facial nerve to the basal turn of the cochlea. In this study, the mean value of this distance was $10.7 \mathrm{~mm}$ and the maximum distance encountered was $12 \mathrm{~mm}$. These values correlate well with the values obtained from cadaveric specimens in our study, which averaged a composite mean of $9.4 \mathrm{~mm}$ and maximum of $10.2 \mathrm{~mm}$ (of note, it is possible the average $1.3 \mathrm{~mm}$ of difference relates to our practice of "blue-lining" the cochlea in this study and measuring to the border of the cochlear membrane). The other two measurements reported in this manuscript were not assessed in the Sennaroglu study or, to the authors' knowledge, in any other studies.

Although the aforementioned studies are useful in regards to approximate localization of the cochlea, they fail to provide the skull base surgeon with a precise method of localization using microsurgical landmarks. The current study describes a method used to predict cochlear location following identification of the extrapolated junction of the GSPN and the facial nerve (-Fig. 4A). Once this junction has been identified, the anterior and medial boundaries of the cochlea can be estimated using the measurements obtained above ( $\mathbf{F i g . ~ 4 B}$ ). Using morphologic and radiologic data obtained in this study, the membranous cochlea was not encountered more than $11 \mathrm{~mm}$ from this extrapolated junction. In the event the skull base surgeon wishes to avoid transgression of the cortical bone of the cochlea, as is almost always the case, this figure should be increased to $12.5 \mathrm{~mm}$, based on previous data estimating the thickness of the cochlear bone at 1.1 to $1.7 \mathrm{~mm}^{11}$

Data from this study obtained from wet bench cadaveric dissection of six heads correlated extremely well with data obtained following dissection of five dry temporal bones (-Table 1). Cadaveric data, in turn, correlated well with radiologic data (-Table 2), with one exception. The average distance of the extrapolated junction of the GSPN and facial nerve to the anterior border of the membranous cochlea was, on average, $2.0 \mathrm{~mm}$ less in the radiologic set. This discrepancy likely relates to difficulty with obtaining this specific measurement in a prefixed axial plane but may also relate to the

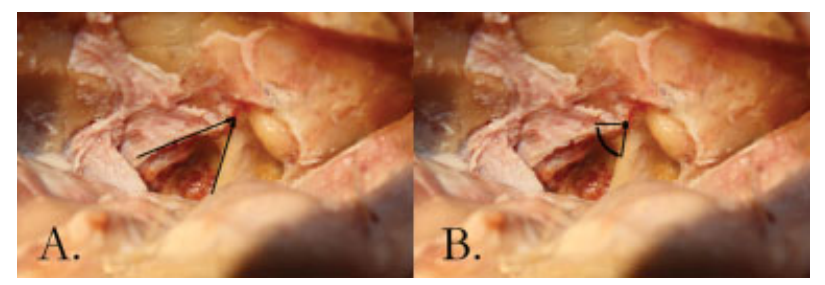

Fig. 4 Method of cochlear localization. (A) Following identification of the greater superficial petrosal nerve (GSPN) and $7 / 8$ complex (arrows), the extrapolated junction of the two structures is estimated (A). The membranous cochlea was encountered not more than $11 \mathrm{~mm}$ from this approximate junction (junction depicted via intersection of two line segments; $B$ ). 
chosen method of estimating the position of the GPSN based on the underlying bony groove-which has not undergone previous, rigorous evaluation. Interestingly, the other two parameters that were measured demonstrated a high level of agreement between radiologic and morphologic data.

Review of the data obtained indicates that a "danger zone" exists within $11 \mathrm{~mm}$ of the extrapolated junction of the GSPN and facial nerve. Drilling of the anteromedial petrous bone within of a radius of $11 \mathrm{~mm}$ from the extrapolated junction of GSPN and facial nerve appears to be associated with a high degree of risk to the membranous cochlea. Drilling outside of a radius of $12.5 \mathrm{~mm}$ from the extrapolated junction of GSPN and facial nerve appears to be associated with a low degree of risk to the bony cochlear apparatus.

In conclusion, our study demonstrates that, when drilling Kawase's rhomboid, it is useful to locate the extrapolated junction of the GSPN and the facial nerve. Drilling of the anteromedial petrous bone outside of a radius of $12.5 \mathrm{~mm}$ from the extrapolated junction of GSPN and facial nerve appears to be associated with a low degree of risk to the cochlear apparatus.

\section{References}

1 Day JD, Fukushima T, Giannotta SL. Microanatomical study of the extradural middle fossa approach to the petroclival and posterior cavernous sinus region: description of the rhomboid construct. Neurosurgery 1994;34:1009-1016, discussion 1016
2 Miller CG, van Loveren HR, Keller JT, Pensak M, el-Kalliny M, Tew JM Jr. Transpetrosal approach: surgical anatomy and technique. Neurosurgery 1993;33:461-469, discussion 469

3 Garcia-Ibanez E, Garcia-Ibanez JL. Middle fossa vestibular neurectomy: a report of 373 cases. Otolaryngol Head Neck Surg 1980; 88:486-490

4 Isolan GR, Krayenbühl N, de Oliveira E, Al-Mefty O. Microsurgical anatomy of the cavernous sinus: measurements of the triangles in and around it. Skull Base 2007;17:357-367

5 Sameshima T, Mastronardi L, Friedman A, Fukushima T. Middle fossa dissection for extended middle fossa and anterior petrosectomy. In: Sameshima T, Mastronardi L, Friedman A, Fukushima T, eds. Fukushima's Microanatomy and Dissection of the Temporal Bone for Surgery of Acoustic Neuroma and Petroclival Meningioma. Raleigh: AF NeuroVideo; 2004

6 Mastronardi L, Sameshima T, Ducati A, De Waele LF, Ferrante L, Fukushima T. Extradural middle fossa approach. Proposal of a learning method: the "rule of two fans." Technical note. Skull Base 2006;16:181-184

7 Dew LA, Shelton C, Harnsberger HR, Thompson BG Jr. Surgical exposure of the petrous internal carotid artery: practical application for skull base surgery. Laryngoscope 1997;107:967-976

8 Rhoton AL Jr. The temporal bone and transtemporal approaches. Neurosurgery 2000;47(3, Suppl):S211-S265

9 Jung SM. JSJ, Ahn T.H. Microanatomical study of the extradural middle fossa approach for preventing cochlear damage. J Korean Neurosurg Soc 2004;36:353-357

10 Sennaroglu L, Slattery WH III. Petrous anatomy for middle fossa approach. Laryngoscope 2003;113:332-342

11 Laurikainen E, Kanninen P, Aho H, Saukko P. The anatomy of the human promontory for laser Doppler flowmetry. Eur Arch Otorhinolaryngol 1997;254:264-268

12 Jackler RK, ed. Atlas of Skull Base Surgery and Neurotology. New York, NY: Thieme; 2008 